\title{
A NOTE ON MULTINOMIAL PROBABILISTIC VALUES*
}

\author{
Francesc Carreras ${ }^{\dagger}$ and María Albina Puente ${ }^{\ddagger}$
}

September 14, 2017

\begin{abstract}
Multinomial values were previously introduced by one of the authors in reliability and extended later to all cooperative games. Here, we present for this subfamily of probabilistic values three new results, previously stated only for binomial semivalues in the literature. They concern the dimension of the subspace spanned by the multinomial values and two characterizations: one, individual, for each multinomial value; another, collective, for the whole subfamily they form. Finally, an application to simple games is provided.
\end{abstract}

Keywords: game theory, cooperative game, Shapley value, probabilistic value, binomial semivalue.

Math. Subj. Class.: 91A12.

\section{Introduction}

Probabilistic values, introduced by Weber [20], constitute a family of values for cooperative games axiomatically characterized by means of linearity, positivity, and the dummy player property. Every probabilistic value allocates, to each player in each game of its domain, a weighted (convex) sum of all marginal contributions of the player in the game. Among probabilistic values, semivalues [10] are characterized by the anonymity property or, equivalently, by the fact that each weighting coefficient depends only on the cardinality of the coalition to which it refers. The Shapley value [19] is the only efficient semivalue, whereas the Banzhaf value [16] is the only semivalue that satisfies the total power property.

In this paper, we focus on a subfamily of probabilistic values called multinomial values. These values were first introduced in reliability by Puente [18] (see also [12]) with the name of "multibinary probabilistic values." They were independently defined by Carreras [3], for simple games onlyi.e., as power indices - , in a study on decisiveness (see also [4]) where they were called "Banzhaf $\alpha$-indices." Later on, Carreras and Puente [6] extended them to all cooperative games and began to use the current term "multinomial". A deep additional study of interesting properties of these values can be found in [8].

The main characteristics of the multinomial values are: (1) each one of them is defined by $n$ parameters ( $n$ being the number of players) and (2) the weighting coefficients of the value are systematically generated in terms of these parameters. We attach to parameter $p_{i}$ the meaning of

\footnotetext{
*This research project was partially supported by funds from the Spanish Ministry of Economy and Competitiveness (MINECO) and from the European Union (FEDER funds) under grant MTM2015-66818-P (MINECO/FEDER).

${ }^{\dagger}$ Universitat Politècnica de Catalunya (UPC), Department of Mathematics, School of Industrial, Aerospace and Audiovisual Engineering of Terrassa, Spain. E-mail: francesc.carreras@upc.edu.

${ }^{\ddagger}$ Universitat Politècnica de Catalunya (UPC), Department of Mathematics, Engineering School of Manresa, Spain. E-mail: m.albina.puente@upc.edu. Corresponding author.
} 
generical tendency of player $i$ to form coalitions, assuming that $p_{i}$ and $p_{j}$ are independent of each other if $i \neq j$. We also assume that $0 \leq p_{i} \leq 1$ for each player $i$ and collect all parameters in a tendency profile $\mathbf{p}=\left(p_{1}, p_{2}, \ldots, p_{n}\right)$.

These tendency profiles can encompass a variety of situations arising from players' attitudes. As is shown in [6], multinomial values offer a flexibility evidently greater than binomial semivalues $[18,12]$ - whose monoparametric condition implies a quite limited capability of analysis of such situations - and, hence, many more possibilities to introduce additional information when evaluating a game. In [14], the multinomial values are used to study the effects of the partnership formation in cooperative games, comparing the joint effect on the involved players with the effect of the alternative alliance formation. In [7], multinomial values are extended to provide the coalitional multinomial probabilistic values, designed to take into account players' attitudes with regard to cooperation. This new family of coalitional values applies to cooperative games with a coalition structure by combining the Shapley value and the multinomial values.

In Fig. 1, we show the position of multinomial values in the framework of probabilistic values.

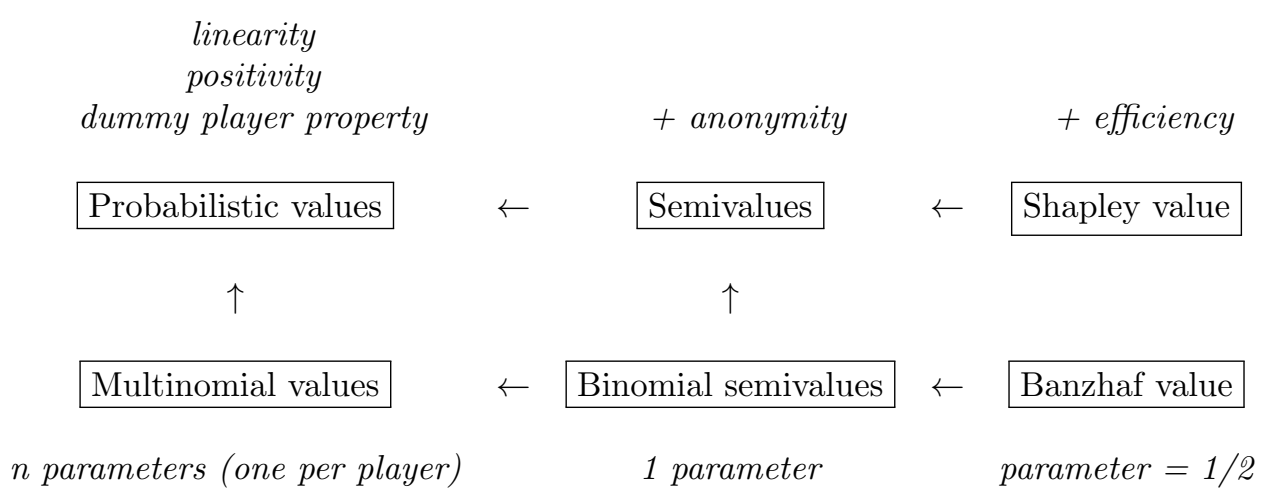

Fig. 1: Inclusion relationships between values and families of values

Simple games constitute an interesting class of cooperative games. Not only as a test bed for cooperative concepts but also for the variety of their interpretations (often far from game theory). In particular, they are frequently applied to describe and analyze collective decisionmaking mechanisms ruled by voting.

In order to fix ideas, let us assume that a single proposal $P$, such as a bill or an amendment, is pitted against the status quo $Q$. Each agent (player) has only two options: voting for $P$ or voting against it and hence for maintaining the status quo. The rules must state the groups of agents (coalitions) which can pass the proposal when voting for it (such a collection of winning coalitions defines a simple game in the set of agents), so abstention is implicitly allowed but it counts for $Q$. Usually, each agent controls a number of votes (weight) and the proposal receives approval if and only if the total weight of the group of agents that vote for it meets or exceeds a given threshold (quota): we then speak of a weighted majority game as a particular case.

In this context, $p_{i}$ may be understood as the probability of each player $i$ to vote for the proposal (and hence $1-p_{i}$ as the probability to vote against or abstain). The multinomial value attached to profile $\mathbf{p}=\left(p_{1}, p_{2}, \ldots, p_{n}\right)$ provides a measure of the relative decision power of each agent.

Example 1.1 The staff of a law firm A is made up of five members, the first three of which are founding members and possess veto right in the decision-making process of the firm. The other two are young interns of the law firm. A new law firm B of the competition wishes to merge with A. Each A member will have to choose among two opposite possibilities: accepting to merge with 
B (option 1) or refusing the offer (option 2). Abstention is not allowed. Each A member has an independent probability of voting for merging. The members of $\mathrm{B}$, who prefer option 1 , want to influence the opinion of the members of A before they take the decision. With this purpose, firm $\mathrm{B}$ will present the merging proposal from a viewpoint very favorable to option 1 . In this situation, (a) which of the A members has more decision power? and (b) if firm B considers the possibility to bribe one of the A members to the extent that he/she votes option 1 with probability 1 , which of them will be the best candidate? The answers will depend on the view held by each A member. The details will be given in Section 5 .

The remainder of this paper is organized as follows. Section 2 includes some basic preliminaries. In Section 3, we recall the notion of multinomial value. Section 4 is devoted to extend three results stated in the previous literature for binomial semivalues only. Thus, we determine the dimension of the subspace spanned by multinomial values and provide two characterizations: one, individual, for each multinomial value; another, collective, for the whole subfamily they form. In all cases the proofs are not straightforward and reveal new features of multinomial values. Finally, in Section 5 we focus on simple games and discuss Example 1.1.

\section{Preliminaries}

Let $N$ be a finite set of players, usually denoted as $N=\{1,2, \ldots, n\}$. A cooperative game in $N$ is a function $v$ that assigns a real number $v(S)$ to each coalition $S \subseteq N$, with $v(\emptyset)=0$. This number is understood as the utility that coalition $S$ can obtain by itself, that is, independently of the remaining players' behavior.

Game $v$ is monotonic if $v(S) \leq v(T)$ when $S \subset T \subseteq N$. Player $i \in N$ is a dummy in game $v$ if $v(S \cup\{i\})=v(S)+v(\{i\})$ for all $S \subseteq N \backslash\{i\}$, and null if, moreover, $v(\{i\})=0$. Players $i, j \in N$ are symmetric in $v$ if $v(S \cup\{i\})=v(S \cup\{j\})$ for all $S \subseteq N \backslash\{i, j\}$.

Endowed with the natural operations for real-valued functions, $v+v^{\prime}$ and $\lambda v$ for all $\lambda \in \mathbb{R}$, the set of all cooperative games in $N$ is a vector space $\mathcal{G}_{N}$. For every nonempty $T \subseteq N$, the unanimity game $u_{T}$ in $N$ is defined by $u_{T}(S)=1$ if $T \subseteq S$ and $u_{T}(S)=0$ otherwise, and it is easily checked that the set of all unanimity games is a basis for $\mathcal{G}_{N}$, so that $\operatorname{dim} \mathcal{G}_{N}=2^{n}-1$. Every permutation $\theta$ of $N$ induces a linear automorphism of $\mathcal{G}_{N}$ given by $(\theta v)(S)=v\left(\theta^{-1} S\right)$ for all $S \subseteq N$ and all $v$.

By a value on $\mathcal{G}_{N}$, we mean a map $g: \mathcal{G}_{N} \rightarrow \mathbb{R}^{n}$, which assigns to every game $v$ a vector $g[v]$ with components $g_{i}[v]$ for all $i \in N$. The total power of value $g$ in $v$ is

$$
\pi^{g}(v)=\sum_{i \in N} g_{i}[v] .
$$

Following Weber's axiomatic definition [20], $\phi: \mathcal{G}_{N} \rightarrow \mathbb{R}^{n}$ is a (group) probabilistic value if it satisfies the following properties:

(i) linearity: $\phi\left[v+v^{\prime}\right]=\phi[v]+\phi\left[v^{\prime}\right]$ and $\phi[\lambda v]=\lambda \phi[v]$ for all $v, v^{\prime} \in \mathcal{G}_{N}$ and $\lambda \in \mathbb{R}$;

(ii) positivity ${ }^{1}$ : if $v$ is monotonic, then $\phi[v] \geq 0$;

(iii) dummy player property: if $i \in N$ is a dummy in game $v$, then $\phi_{i}[v]=v(\{i\})$.

There is an interesting characterization of the probabilistic values, also in [20]: (a) given a set of $n 2^{n-1}$ weighting coefficients $P=\left\{p_{S}^{i}: i \in N, S \subseteq N \backslash\{i\}\right\}$, such that

$$
\text { all } p_{S}^{i} \geq 0 \quad \text { and } \quad \sum_{S \subseteq N \backslash\{i\}} p_{S}^{i}=1 \text { for each } i,
$$

\footnotetext{
${ }^{1}$ In [20] this property is called monotonicity, but we prefer to call to it positivity as in [10].
} 
the expression

$$
\phi_{i}[v]=\sum_{S \subseteq N \backslash\{i\}} p_{S}^{i}[v(S \cup\{i\})-v(S)] \quad \text { for all } i \in N \text { and } v \in \mathcal{G}_{N}
$$

defines a probabilistic value $\phi$ on $\mathcal{G}_{N} ;(\mathrm{b})$ conversely, every probabilistic value can be obtained in this manner; (c) the correspondence given by $P \mapsto \phi$ is one-to-one. Thus, the payoff that a probabilistic value allocates to every player in any game is a convex sum of the marginal contributions of the player in the game. We quote from [20]:

"Let player $i$ view his participation in a game $v$ as consisting merely of joining some coalition $S$ and then receiving as a reward his marginal contribution to the coalition. If $p_{S}^{i}$ is the probability that he joins coalition $S$, then $\phi_{i}[v]$ is his expected payoff from the game."

All probabilistic values are linear, and therefore it is interesting to know their action on unanimity games because these form a basis of the space of games. It is as follows:

$$
\phi_{i}\left[u_{T}\right]=\sum_{\substack{S \subseteq N \backslash\{i\}: \\ T \backslash\{i\} \subseteq S}} p_{S}^{i} \quad \text { if } i \in T \quad \text { and } \quad \phi_{i}\left[u_{T}\right]=0 \quad \text { otherwise. }
$$

Among probabilistic values, semivalues, introduced by Dubey et al. [10], are characterized by the anonymity property: $\phi_{\theta i}[\theta v]=\phi_{i}[v]$ for all $i \in N, v \in \mathcal{G}_{N}$ and $\theta$. This is equivalent [20] to saying that, if $n=|N|$, there is a vector $\left\{p_{s}\right\}_{s=0}^{n-1}$ such that $p_{S}^{i}=p_{s}$ for all $i \in N$ and all $S \subseteq N \backslash\{i\}$, where $s=|S|$, so all coalitions of a given size share a common weight and Eq. (3) reduces to

$$
\phi_{i}[v]=\sum_{S \subseteq N \backslash\{i\}} p_{s}[v(S \cup\{i\})-v(S)] \quad \text { for all } i \in N \text { and } v \in \mathcal{G}_{N} .
$$

The weighting coefficients $\left\{p_{s}\right\}_{s=0}^{n-1}$ of any semivalue $\phi$ satisfy two characteristic conditions, derived from Eq. (2): each $p_{s} \geq 0$ and $\sum_{s=0}^{n-1}\left(\begin{array}{c}n-1 \\ s\end{array}\right) p_{s}=1$.

Among semivalues, the Shapley value [19], denoted here by $\varphi$ and defined by $p_{s}=1 /\left(\begin{array}{c}n-1 \\ s\end{array}\right) n$ for all $s$, is the only efficient semivalue, in the sense that $\pi^{\varphi}(v)=\sum_{i \in N} \varphi_{i}[v]=v(N)$ for every $v \in \mathcal{G}_{N}$. The Banzhaf value [16], denoted here by $\beta$ and defined by $p_{s}=1 / 2^{n-1}$ for all $s$, is the only semivalue satisfying the total power property:

$$
\pi^{\beta}(v)=\sum_{i \in N} \beta_{i}[v]=\frac{1}{2^{n-1}} \sum_{S \subseteq N} \sum_{i \notin S}[v(S \cup\{i\})-v(S)] \quad \text { for every } v \in \mathcal{G}_{N} .
$$

The Banzhaf value is also the only semivalue with constant weighting coefficients.

Finally, the multilinear extension of a game $v \in \mathcal{G}_{N}$, introduced by Owen [15], is the real-valued function defined in $\mathbb{R}^{n}$ by

$$
f\left(x_{1}, x_{2}, \ldots, x_{n}\right)=\sum_{S \subseteq N} \prod_{i \in S} x_{i} \prod_{j \in N \backslash S}\left(1-x_{j}\right) v(S) .
$$

Both the Shapley and Banzhaf values of any game $v$ can be obtained from its multilinear extension. Indeed, $\varphi[v]$ can be calculated by integrating the partial derivatives of the multilinear extension of the game along the main diagonal $x_{1}=x_{2}=\cdots=x_{n}$ of the cube $[0,1]^{n}[15]$, while the partial derivatives of that multilinear extension, evaluated at point $(1 / 2,1 / 2, \ldots, 1 / 2)$, give $\beta[v][16]$. 


\section{Multinomial values}

We recall here the definition of multinomial values for cooperative games established in [6].

Definition 3.1 Let $N=\{1,2, \ldots, n\}$ be the set of players and let $\mathbf{p} \in[0,1]^{n}$, that is, $\mathbf{p}=$ $\left(p_{1}, p_{2}, \ldots, p_{n}\right)$ with $0 \leq p_{i} \leq 1$ for $i=1,2, \ldots, n$, be a given profile. Then, the coefficients

$$
p_{S}^{i}=\prod_{j \in S} p_{j} \prod_{\substack{k \in N \backslash S: \\ k \neq i}}\left(1-p_{k}\right) \quad \text { for all } i \in N \text { and } S \subseteq N \backslash\{i\}
$$

(the empty product, arising if $S=\emptyset$ or $S=N \backslash\{i\}$, is taken to be 1) define a probabilistic value on $\mathcal{G}_{N}$, called the $\mathbf{p}-$ multinomial probabilistic value and denoted as $\lambda^{\mathbf{p}}$. Its action is given by

$$
\lambda_{i}^{\mathbf{p}}[v]=\sum_{S \subseteq N \backslash\{i\}}\left[\prod_{j \in S} p_{j} \prod_{\substack{k \in N \backslash S: \\ k \neq i}}\left(1-p_{k}\right)\right][v(S \cup\{i\})-v(S)] \quad \text { for all } i \in N \text { and } v \in \mathcal{G}_{N} .
$$

As was announced in Section 1, we attach to $p_{i}$ the meaning of generic tendency of player $i$ to form coalitions, and thus we say that $\mathbf{p}$ is a tendency profile on $N$. According to Eq. (6), coefficient $p_{S}^{i}$, the probability of $i$ to join $S$, will depend on the positive tendencies of the members of $S$ to form coalitions and also on the negative tendencies in this sense of the outside players, i.e., the members of $N \backslash(S \cup\{i\})$. Thus, neither $p_{S}^{i}$ nor $\lambda_{i}^{\mathbf{p}}[v]$ depend on $p_{i}$ but on all $p_{j}$ for $j \neq i$.

Remarks 3.2 (a) For example, for $n=2$ we have $\mathbf{p}=\left(p_{1}, p_{2}\right)$ and, if $i \neq j$,

$$
\lambda_{i}^{\mathbf{p}}[v]=\left(1-p_{j}\right)[v(\{i\})-v(\emptyset)]+p_{j}[v(N)-v(\{j\})] .
$$

Hence, the allocation given by $\lambda^{\mathbf{p}}$ to player $i$ does not depend on $p_{i}$ but only on $p_{j}$. If player $j$ is not greatly interested in cooperating (say, $p_{j}$ tends to 0 ), player $i$ 's allocation will tend to his individual utility $v(\{i\})$. Instead, if player $j$ is highly interested in cooperating (say, $p_{j}$ tends to 1 ), player $i$ 's allocation will tend to his marginal contribution to the grand coalition $v(N)-v(\{j\})$.

(b) For $n=3$ we have $\mathbf{p}=\left(p_{1}, p_{2}, p_{3}\right)$ and, if $i, j, k$ are distinct players,

$$
\lambda_{i}^{\mathbf{p}}[v]=\bar{p}_{j} \bar{p}_{k} v(\{i\})+p_{j} \bar{p}_{k}[v(\{i, j\})-v(\{j\})]+\bar{p}_{j} p_{k}[v(\{i, k\})-v(\{k\})]+p_{j} p_{k}[v(N)-v(\{j, k\})]
$$

(where $\bar{p}_{j}=1-p_{j}$ and $\bar{p}_{k}=1-p_{k}$ for shortness). The comments here would be similar to those made in (a).

(c) A first extreme case arises whenever $p_{j}=0$ for some player $j$. Then, it is easy to see that the remaining players obtain the same payoff as in game $v^{-\{j\}}$, the restriction of $v$ to $N \backslash\{j\}$, with profile $p^{-\{j\}}=\left(p_{1}, p_{2}, \ldots, \hat{p}_{j}, \ldots, p_{n}\right)$, that is, they bargain among themselves disregarding player $j$, who obtains the payoff (independent of $p_{j}$ ) in the original game $v$. A second extreme case arises whenever $p_{j}=1$ for some player $j$. Then, it is easy to see that the remaining players obtain the same payoff as in game $v^{j}$, a new game in $N$ where $v^{j}(S)=v(S)$ if $j \in S$ and $v^{j}(S)=0$ otherwise, with profile $p=\left(p_{1}, p_{2}, \ldots, p_{j}=1, \ldots, p_{n}\right)$, that is, they bargain in a new game where only coalitions containing $j$ matter, whereas $j$ obtains the payoff in the original game $v$.

(d) It is easy to check that the action of $\lambda^{\mathbf{p}}$ on a unanimity game $u_{T}$ is given by

$$
\lambda_{i}^{\mathbf{p}}\left[u_{T}\right]=\prod_{j \in T \backslash\{i\}} p_{j} \quad \text { if } i \in T \quad \text { and } \quad \lambda_{i}^{\mathbf{p}}\left[u_{T}\right]=0 \quad \text { otherwise. }
$$

(If $T=\{i\}$ then $\lambda_{i}^{\mathbf{p}}\left[u_{T}\right]=1$, again by the convention, established in Definition 3.1, that any empty product equals 1.) Using Eq. (8), it readily follows that, for $n \geq 2, \mathbf{p} \neq \mathbf{p}^{\prime}$ implies $\lambda^{\mathbf{p}} \neq \lambda^{\mathbf{p}^{\prime}}$ (if $n=1$ all profiles give rise to a unique multinomial value). 
(e) Whenever, in particular, $p_{1}=p_{2}=\cdots=p_{n}=q$ for some $q \in[0,1]$, coefficients $p_{S}^{i}$ reduce, for all $i \in N$, to

$$
p_{S}^{i}=p_{s}=q^{s}(1-q)^{n-s-1} \quad \text { for } s=0,1, \ldots, n-1,
$$

where $s=|S|$ and, by convention, $0^{0}=1$ for $q=0$ and $q=1$. These coefficients $\left\{p_{s}\right\}_{s=0}^{n-1}$ define the $q$-binomial semivalue $\psi^{q}$ introduced in [18] and, obviously, $\lambda^{\mathbf{p}}=\psi^{q}$. If, moreover, $q=1 / 2$, then, we obtain $\psi^{1 / 2}=\beta$, the Banzhaf value.

(f) The multilinear extension technique adapts well to all binomial semivalues $[18,1]$ and even to any multinomial value $\lambda^{\mathbf{p}}$ (cf. $\left.[18,12]\right)$ : if $f$ is the multilinear extension of game $v \in \mathcal{G}_{N}$ then

$$
\lambda_{i}^{\mathbf{p}}[v]=\frac{\partial f}{\partial x_{i}}\left(p_{1}, p_{2}, \ldots, p_{n}\right) \quad \text { for all } i \in N .
$$

\section{Three theoretical results}

We devote this section to extending three results stated in the previous literature on binomial semivalues. In all cases, the extension is not straightforward and reveals new features of multinomial values. We assume $n \geq 2$ because for $n=1$ all is trivial.

\subsection{About dimensions}

Let $\mathcal{L}\left(\mathcal{G}_{N}, \mathbb{R}^{n}\right)$ denote the space of all linear maps from $\mathcal{G}_{N}$ to $\mathbb{R}^{n}$, which includes most values studied in the literature. It is clear that $\operatorname{dim} \mathcal{L}\left(\mathcal{G}_{N}, \mathbb{R}^{n}\right)=n\left(2^{n}-1\right)$. Let $\mathcal{B S}\left(\mathcal{G}_{N}, \mathbb{R}^{n}\right)$ denote the subspace spanned by binomial semivalues. As is shown in [12], $\operatorname{dim} \mathcal{B S}\left(\mathcal{G}_{N}, \mathbb{R}^{n}\right)=n$ and, moreover, $\mathcal{B S}\left(\mathcal{G}_{N}, \mathbb{R}^{n}\right)$ coincides with the subspace spanned by all semivalues, and any $n$ different binomial semivalues $\psi^{q_{1}}, \psi^{q_{2}}, \ldots, \psi^{q_{n}}$ form a basis.

Let $\mathcal{M V}\left(\mathcal{G}_{N}, \mathbb{R}^{n}\right)$ denote the subspace spanned by multinomial values. Of course, $\mathcal{B S}\left(\mathcal{G}_{N}, \mathbb{R}^{n}\right) \subseteq$ $\mathcal{M V}\left(\mathcal{G}_{N}, \mathbb{R}^{n}\right)$. We shall determine $\operatorname{dim} \mathcal{M} V\left(\mathcal{G}_{N}, \mathbb{R}^{n}\right)$ and provide a basis for this subspace. To this end, an auxiliar notion will be useful.

Definition 4.1 A value $g$ on $\mathcal{G}_{N}$ satisfies the property of neutrality (for unanimity games) if, for each $T \subseteq N$ with $0 \leq|T| \leq n-2$,

$$
g_{i}\left[u_{T \cup\{i\}}\right]=g_{j}\left[u_{T \cup\{j\}}\right] \quad \text { for any } i, j \notin T \text {. }
$$

This property is a sort of symmetry condition for players restricted to unanimity games. It is satisfied by any multinomial value ${ }^{2}$ since, by Remark $3.2(\mathrm{~d})$, we have

$$
\lambda_{i}^{\mathbf{p}}\left[u_{T \cup\{i\}}\right]=\prod_{k \in T} p_{k}=\lambda_{j}^{\mathbf{p}}\left[u_{T \cup\{j\}}\right] .
$$

Theorem 4.2 Let $\mathcal{M} V\left(\mathcal{G}_{N}, \mathbb{R}^{n}\right)$ be the subspace spanned by multinomial values within the space $\mathcal{L}\left(\mathcal{G}_{N}, \mathbb{R}^{n}\right)$ of linear maps. Then:

(a) $\operatorname{dim} \mathcal{M V}\left(\mathcal{G}_{N}, \mathbb{R}^{n}\right)=2^{n}-1$.

(b) If, for each $S \subset N$ (including $S=\emptyset$ ), a profile $\mathbf{p}^{S}=\left(p_{1}^{S}, p_{2}^{S}, \ldots, p_{n}^{S}\right)$ is defined by

$$
p_{i}^{S}= \begin{cases}1 & \text { if } i \in S, \\ 0 & \text { otherwise }\end{cases}
$$

the family of corresponding multinomial values $\left\{\lambda^{\mathbf{p}^{S}}: S \subset N\right\}$ is a basis of $\mathcal{M} V\left(\mathcal{G}_{N}, \mathbb{R}^{n}\right)$.

\footnotetext{
${ }^{2}$ In particular, all binomial semivalues, but also the Shapley value, satisfy this property.
} 
Proof. (a) Every $\phi \in \mathcal{M V}\left(\mathcal{G}_{N}, \mathbb{R}^{n}\right)$ is of the form

$$
\phi=\alpha_{1} \lambda^{\mathbf{p}^{1}}+\alpha_{2} \lambda^{\mathbf{p}^{2}}+\cdots+\alpha_{k} \lambda^{\mathbf{p}^{k}},
$$

where $\alpha_{1}, \alpha_{2}, \ldots, \alpha_{k} \in \mathbb{R}$ and $\lambda^{\mathbf{p}^{1}}, \lambda^{\mathbf{p}^{2}}, \ldots, \lambda^{\mathbf{p}^{k}}$ are multinomial values. Then, $\phi$ satisfies linearity, the dummy player property for null players, and neutrality. As every game is a linear combination of unanimity games, by linearity it follows that $\phi$ is completely determined by its action on unanimity games. Moreover, the null player property implies that $\phi_{i}\left[u_{T}\right]=0$ if $i \notin T$. Then, at this stage it is clear that $\phi$ is determined by $\sum_{t=1}^{n} t\left(\begin{array}{c}n \\ t\end{array}\right)=n 2^{n-1}$ linear parameters, namely, the allocations $\phi_{i}\left[u_{T}\right]$ for all nonempty $T \subseteq N$ and all $i \in T$.

It remains using neutrality to reduce the number of parameters. For each cardinality $t$ such that $0 \leq t \leq n-1$, there are $\left(\begin{array}{l}n \\ t\end{array}\right)$ coalitions $T$ such that $|T|=t$. If we add to each such $T$ each $i \notin T$, then we get for each $t$ a list of $\left(\begin{array}{c}n \\ t\end{array}\right)(n-t)$ coalitions of the form $T \cup\{i\}$, and hence of cardinality $t+1$, but each one is repeated $t+1$ times. Then, the set of all $\phi_{j}\left[u_{T \cup\{i\}}\right]$ such that $0 \leq|T| \leq n-1, i \notin T$ and $j \in T \cup\{i\}$ coincides with the set of linear parameters $(t+1$ for each $T \cup\{i\})$ mentioned at the end of the above paragraph. Therefore, neutrality applies and, for each $T, n-t$ parameters are reduced to just one of them: e.g., we have

$$
\phi_{1}\left[u_{\{1\}}\right]=\phi_{2}\left[u_{\{2\}}\right]=\cdots=\phi_{n}\left[u_{\{n\}}\right] \quad(\text { for } T=\emptyset) .
$$

Thus, in all, the number of linear parameters necessary to define $\phi$ has been reduced to $\sum_{t=0}^{n-1}\left(\begin{array}{l}n \\ t\end{array}\right)=2^{n}-1$, and hence $\operatorname{dim} \mathcal{M} V\left(\mathcal{G}_{N}, \mathbb{R}^{n}\right) \leq 2^{n}-1$.

(b) To complete the proof, it will suffice to show that the $2^{n}-1$ multinomial values introduced in the statement of the theorem are linearly independent. This will imply that $\operatorname{dim} \mathcal{M} V\left(\mathcal{G}_{N}, \mathbb{R}^{n}\right)=$ $2^{n}-1$ and these values will form a basis of $\mathcal{M} V\left(\mathcal{G}_{N}, \mathbb{R}^{n}\right)$. Assume that, for some set of real numbers $\left\{\alpha_{S}: S \subset N\right\}$,

$$
\phi=\sum_{S \subset N} \alpha_{S} \lambda^{\mathbf{p}^{S}}=0
$$

By applying this linear combination only to selected unanimity games and players, we obtain a homogeneous system of just $2^{n}-1$ linear equations with $2^{n}-1$ unknowns, namely, the coefficients $\alpha_{S}$ of Eq. (9). The selection is very simple: for every coalition $S \subset N$ we choose one external player $i$. Then we compute $\phi$ for this player in game $u_{S \cup\{i\}}$. In all, we have $2^{n}-1$ equations.

Let us consider the case $n=4$, which clearly illustrates the general (systematic) selection procedure that works for any $n$. Table 1 below shows the selected games and players to which we apply all multinomial values $\lambda^{\mathbf{p}^{S}}$. The ordering by which we select a coalition $S$, add to it an external player $i$, and consider in the first column the unanimity game $u_{S \cup\{i\}}$, coincides with the ordering of coalitions in the first row of the table, and we always add the player with the lowest denomination in the natural ordering (indicated in the second column). At the right of this table we find the matrix of coefficients of the linear system.

Since (here and for all $n$ ) we obtain an upper triangular matrix with 1's on the diagonal, the only solution of the homogeneous system is obviously

$$
\alpha_{S}=0 \text { for all } S \subset N
$$

and hence the $2^{n}-1$ multinomial values $\lambda^{\mathbf{p}^{S}}$ are linearly independent and constitute a (natural) basis for $\mathcal{M V}\left(\mathcal{G}_{N}, \mathbb{R}^{n}\right)$. Of course, in some cases there may exist different ways to select the external player, but neutrality guarantees that all choices give rise to the same conclusion. 


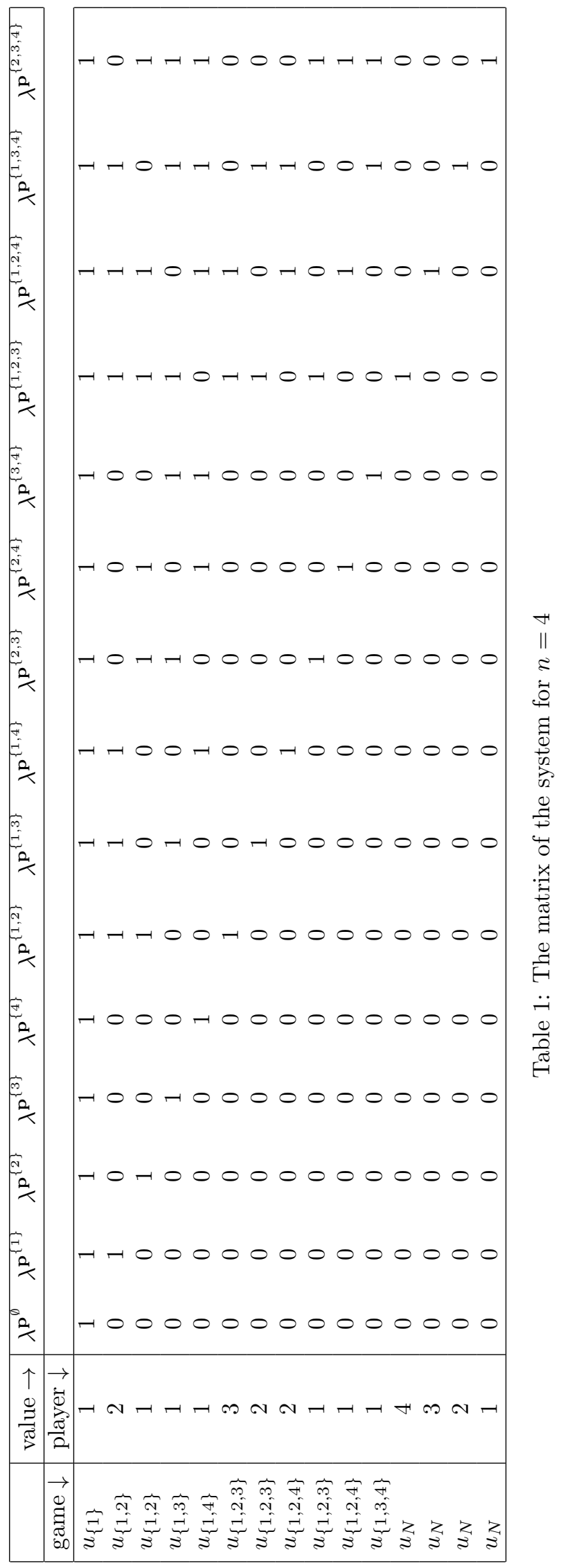


Remarks 4.3 (a) The difference between $n=\operatorname{dim} \mathcal{B S}\left(\mathcal{G}_{N}, \mathbb{R}^{n}\right)$ and $2^{n}-1=\operatorname{dim} \mathcal{M} V\left(\mathcal{G}_{N}, \mathbb{R}^{n}\right)$ reflects the much greater versatility of multinomial values. Incidentally, notice that the Shapley value belongs to $\mathcal{B S}\left(\mathcal{G}_{N}, \mathbb{R}^{n}\right)$, and hence, to $\mathcal{M} V\left(\mathcal{G}_{N}, \mathbb{R}^{n}\right)$. Thus, the Shapley value can be written as a unique linear combination of any set of $n$ different binomial semivalues and also of any basis of $\mathcal{M V}\left(\mathcal{G}_{N}, \mathbb{R}^{n}\right)$, e.g., the basis given in Theorem 4.2. For $n=2$ we find

$$
\varphi=\frac{1}{2} \lambda^{\mathbf{p}^{\{1\}}}+\frac{1}{2} \lambda^{\mathbf{p}^{\{2\}}}
$$

and, for $n=3$,

$$
\varphi=\frac{1}{2} \lambda^{\mathbf{p}^{\emptyset}}-\frac{1}{6} \lambda^{\mathbf{p}^{\{1\}}}-\frac{1}{6} \lambda^{\mathbf{p}^{\{2\}}}-\frac{1}{6} \lambda^{\mathbf{p}^{\{3\}}}+\frac{1}{3} \lambda^{\mathbf{p}^{\{1,2\}}}+\frac{1}{3} \lambda^{\mathbf{p}^{\{1,3\}}}+\frac{1}{3} \lambda^{\mathbf{p}^{\{2,3\}}} .
$$

(b) The property that any family of $n$ different binomial semivalues is a basis of $\mathcal{B S}\left(\mathcal{G}_{N}, \mathbb{R}^{n}\right)$ does not extend to $\mathcal{M} V\left(\mathcal{G}_{N}, \mathbb{R}^{n}\right)$. In effect, e.g for $n=2$, profiles $\mathbf{p}^{\alpha}=(0, \alpha), \mathbf{p}^{\beta}=(0, \beta)$ and $\mathbf{p}^{\gamma}=(0, \gamma)$, with $\alpha, \beta, \gamma$ different from each other, define different multinomial values according to Remark 3.2(d), but it is not difficult to check that they are not linearly independent.

(c) The property that the subspace spanned by all semivalues coincides with the subspace $\mathcal{B S}\left(\mathcal{G}_{N}, \mathbb{R}^{n}\right)$ does not extend to the multinomial level. The subspace spanned by all probabilistic values coincides with $\mathcal{M} V\left(\mathcal{G}_{N}, \mathbb{R}^{n}\right)$ for $n=2$, but not for $n \geq 3$.

(d) However, using a standard basis of $\mathcal{L}\left(\mathcal{G}_{N}, \mathbb{R}^{n}\right)$ to discuss this question, we have arrived to an interesting conclusion: (a) for any $n$, a probabilistic value belongs to $\mathcal{M} V\left(\mathcal{G}_{N}, \mathbb{R}^{n}\right)$ if and only if it satisfies neutrality. The Shapley value is a good example. More generally, (b) a linear value belongs to $\mathcal{M V}\left(\mathcal{G}_{N}, \mathbb{R}^{n}\right)$ if and only if it satisfies neutrality and the null player property. Finally, (c) a value belongs to $\mathcal{M} V\left(\mathcal{G}_{N}, \mathbb{R}^{n}\right)$ if and only if it satisfies linearity, neutrality and the null player property. We omit the details.

\subsection{Individual characterization of each multinomial value}

The notion of total power given by Eq. (1) has proven to be useful in the absence of efficiency. The total power property of the Banzhaf value given by Eq. (5) was the natural substitute of efficiency in the well-known axiomatic characterizations of this value reached in [9] and [11]. It was extended to all binomial semivalues in [5], giving rise to the $q$-binomial total power property:

$$
\pi^{\psi^{q}}(v)=\sum_{i \in N} \psi_{i}^{q}[v]=\sum_{S \subseteq N} q^{s}(1-q)^{n-s-1} \sum_{i \notin S}[v(S \cup\{i\})-v(S)] \quad \text { for every } v \in \mathcal{G}_{N} .
$$

For each $q \in[0,1]$, this property characterizes the $q$-binomial semivalue $\psi^{q}$ among semivalues, and this characterization can be alternatively stated as follows: if $\psi$ is a semivalue such that $\pi^{\psi}(v)=\pi^{\psi^{q}}(v)$ for all $v \in \mathcal{G}_{N}$, then $\psi=\psi^{q}$. The natural extension of the property to probabilistic values must be carried out in the following terms.

Definition 4.4 Let $\mathbf{p} \in[0,1]^{n}$ be a profile on $N$. A (probabilistic or not) value $g$ on $\mathcal{G}_{N}$ satisfies the $\mathbf{p}$-multinomial total power property if, for all $v \in \mathcal{G}_{N}$,

$$
\pi^{g}(v)=\sum_{i \in N} g_{i}[v]=\sum_{S \subseteq N} \sum_{i \notin S} \prod_{j \in S} p_{j} \prod_{\substack{k \in N \backslash S: \\ k \neq i}}\left(1-p_{k}\right)[v(S \cup\{i\})-v(S)] .
$$

However, this property, which is clearly equivalent to $\pi^{g}(v)=\pi^{\lambda^{\mathrm{p}}}(v)$, and hence, obviously satisfied by the $\mathbf{p}$-multinomial value $\lambda^{\mathbf{p}}$, does not characterize this value within the class of probabilistic values. Indeed, it is easy to see, e.g. for $n=2$ and using Eqs. (4) and (8), that in general 
not only $\lambda^{\mathbf{p}}$ but also infinitely many probabilistic values $g$ satisfy Eq. (10) for a given $\mathbf{p}$. And also that the only exceptions correspond, for $n=2$, to $\mathbf{p}=(0,0)$ and $\mathbf{p}=(1,1)$, in which cases we get uniqueness and find, respectively, the dictatorial and the marginal index, binomial semivalues introduced in [17] and defined by $\psi_{i}^{0}[v]=v(\{i\})$ and $\psi_{i}^{1}[v]=v(N)-v(N \backslash\{i\})$ for all $i$ and $v$.

Therefore, we need to introduce a second property in order to characterize each $\lambda^{\mathbf{p}}$ within the class of probabilistic values. The reader will notice that, due to anonymity, this property holds for all binomial semivalues (where $p_{i}=q=p_{j}$ for all $i, j \in N$ ), and hence it was irrelevant for them.

Definition 4.5 Let $\mathbf{p} \in[0,1]^{n}$ be a profile on $N$. A value $g$ on $\mathcal{G}_{N}$ satisfies the property of $\mathbf{p}$-weighted payoffs for unanimity games if, for every nonempty $T \subseteq N$,

$$
p_{i} g_{i}\left[u_{T}\right]=p_{j} g_{j}\left[u_{T}\right] \quad \text { for all } i, j \in T .
$$

This property means that the symmetry between all members of $T$ in the unanimity game $u_{T}$ may well be lost when using the value. More precisely: (a) for players $i, j$ such that $p_{i}$ and $p_{j}$ do not vanish (i.e., $p_{i}, p_{j}>0$ ), the payoff to each such player in $u_{T}$ is inversely proportional to his tendency; and (b) if $p_{i}=0$ then any other player $j \in T$ with positive tendency obtains 0 in $u_{T}$. By using Eq. (8), it is clear that each $\lambda^{\mathbf{p}}$ satisfies this property (with regard to profile $\mathbf{p}$ ), which becomes an important characteristic of this value.

Theorem 4.6 (Characterization of each $\mathbf{p}-$ multinomial value). Let $\mathbf{p}$ be a profile on $N$. Then, the unique probabilistic value on $\mathcal{G}_{N}$ that satisfies the $\mathbf{p}$-multinomial total power property and the property of $\mathbf{p}$-weighted payoffs for unanimity games is the multinomial value $\lambda^{\mathbf{p}}$.

Proof. (Existence) As was mentioned after Definitions 4.4 and 4.5, respectively, the multinomial value $\lambda^{\mathbf{P}}$ satisfies both properties.

(Uniqueness) Using linearity and the fact that the unanimity games form a basis of $\mathcal{G}_{N}$, it suffices to prove that any probabilistic value $\phi$ satisfying both properties with respect to the given profile $\mathbf{p}$ is uniquely determined on the unanimity game $u_{T}$ for each nonempty $T \subseteq N$.

By the dummy player property, $\phi_{i}\left[u_{T}\right]=0$ for all $i \notin T$. If $|T|=1$, set $T=\{i\}$. From the $\mathbf{p}-$ multinomial total power property it follows that $\phi_{i}\left[u_{\{i\}}\right]=1$ since $\lambda_{i}^{\mathbf{p}}\left[u_{\{i\}}\right]=1$ by the convention mentioned after Eq. (8).

Now, let $|T| \geq 2$. We first consider the case of a positive profile, that is, a profile $\mathbf{p}$ with each $p_{i}>0$. If $T=\left\{i_{1}, i_{2}, \ldots, i_{t}\right\}$, with $t=|T|$, by the property of $\mathbf{p}$-weighted payoffs for unanimity games it follows that

$$
p_{i_{1}} \phi_{i_{1}}\left[u_{T}\right]=p_{i_{2}} \phi_{i_{2}}\left[u_{T}\right]=\cdots=p_{i_{t}} \phi_{i_{t}}\left[u_{T}\right]=c_{T},
$$

where $c_{T}$ is a constant (depending only on $\phi$ and $\mathbf{p}$ ). Thus, $\phi_{i}\left[u_{T}\right]=c_{T} / p_{i}$ for each $i \in T$ and, using again the $\mathbf{p}$-multinomial total power property, it follows that

$$
c_{T} \sum_{j \in T} \frac{1}{p_{j}}=\sum_{j \in T} \lambda_{j}^{\mathbf{p}}\left[u_{T}\right]
$$

and therefore, for each $i \in T$,

$$
\phi_{i}\left[u_{T}\right]=\frac{\sum_{j \in T} \lambda_{j}^{\mathbf{p}}\left[u_{T}\right]}{p_{i} \sum_{j \in T} \frac{1}{p_{j}}}=\frac{\sum_{j \in T} \prod_{\substack{k \in T \\ k \neq j}} p_{k}}{p_{i} \sum_{j \in T} \frac{1}{p_{j}}}=\prod_{\substack{k \in T: \\ k \neq i}} p_{k}=\lambda_{i}^{\mathbf{p}}\left[u_{T}\right] .
$$


This completes the proof that $\phi$ is determined (and coincides, necessarily, with $\lambda^{\mathbf{p}}$ ) on each $u_{T}$ when the profile is positive.

Now, let $\mathbf{p}$ be a non-positive profile. Let $R=\left\{i \in N: p_{i}=0\right\}$ and $S=\left\{i \in N: p_{i} \neq 0\right\}$. By assuming $R, S \neq \emptyset$ we disregard the positive profile case, and the dictatorial case $(\mathbf{p}=0)$ where uniqueness clearly holds. Then, two cases arise for $T$ :

(a) $T \cap R=\emptyset$. Then, all $\phi_{i}\left[u_{T}\right]$ for $i \in T$ are determined as for positive profiles.

(b) $T \cap R \neq \emptyset$. Here, using the property of $\mathbf{p}$-weighted payoffs for unanimity games, it follows that $\phi_{i}\left[u_{T}\right]=0$ for all $i \in T \backslash R$. It remains to consider $\phi_{j}\left[u_{T}\right]$ for players $j \in T \cap R$. If $T \cap R=\{j\}$ then $\phi_{j}\left[u_{T}\right]$ is determined, as it equals $\prod_{k \in T \backslash\{j\}} p_{k}$ by the $\mathbf{p}$-multinomial total power property. If $|T \cap R| \geq 2$ then

$$
\sum_{j \in T \cap R} \phi_{j}\left[u_{T}\right]=0
$$

once again by the $\mathbf{p}$-multinomial total power property. However, from the positivity of $\phi$ it follows that $\phi_{j}\left[u_{T}\right]=0$ for all $j \in T \cap R$. Hence, also in the case of a non-positive profile, $\phi$ is determined on $u_{T} \cdot{ }^{3}$

Remark 4.7 Looking at the proof of Theorem 4.6, it is clear that our characterization is adjusted if one wishes to consider any profile (positive or not), since we apply all conditions required to the generic value $\phi$ : being a probabilistic value (linearity, positivity, and the dummy player property) and the additional properties referred to a given profile $\mathbf{p}$, that is, the $\mathbf{p}$-multinomial total power property, and the property of $\mathbf{p}$-weighted payoffs for unanimity games. Nevertheless, if one is interested in positive profiles only, we could state the characterization within the wider set of linear values satisfying the dummy player property, since the positivity of the value (which leads us back to probabilistic values) is used only at the end of the non-positive profile case.

Example 4.8 Theorem 4.6 can be used to verify whether a given probabilistic value is, in fact, a multinomial value. Let $n=3$ and $\phi$ be the probabilistic value defined by the weighting coefficients

$$
\begin{array}{llll}
p_{\emptyset}^{1}=0, & p_{\{2\}}^{1}=0, & p_{\{3\}}^{1}=0.2, & p_{\{2,3\}}^{1}=0.8, \\
p_{\emptyset}^{2}=0, & p_{\{1\}}^{2}=0, & p_{\{3\}}^{2}=0.7, & p_{\{1,3\}}^{2}=0.3, \\
p_{\emptyset}^{3}=0.14, & p_{\{1\}}^{3}=0.06, & p_{\{2\}}^{3}=0.56, & p_{\{1,2\}}^{3}=0.24 .
\end{array}
$$

In principle, an obvious problem seems to be which profile should be used to check the properties. There are infinitely many possibilities to choose from. However, the property of $\mathbf{p}$-weighted payoffs for unanimity games will be very helpful to this end. Indeed, letting for this instance $\mathbf{p}=\left(p_{1}, p_{2}, p_{3}\right)$, we easily find that the property holds if and only if

$$
\frac{p_{1}}{0.3}=\frac{p_{2}}{0.8}=\frac{p_{3}}{1} .
$$

\footnotetext{
${ }^{3}$ The reader might well like to see this argument illustrated for, say, $n=4$. Let e.g. $\mathbf{p}=\left(p_{1}, p_{2}, 0,0\right)$ with $p_{1}, p_{2}>0$. For singletons, we have $\phi_{i}\left[u_{\{j\}}\right]=1$ if $i=j$ and 0 otherwise. For any other nonempty $T, \phi_{i}\left[u_{T}\right]=0$ if $i \notin T$. Coalition $\{1,2\}$ is the only of type $(\mathrm{a})$, and we get $\phi_{i}\left[u_{\{1,2\}}\right]=p_{j}$ if $\{i, j\}=\{1,2\}$. Next, for coalitions $\{1,3\}$, $\{1,4\},\{2,3\},\{2,4\},\{1,2,3\}$ and $\{1,2,4\}$ we have $\phi_{i}\left[u_{T}\right]=0$ if $i=1,2$. Then, from $\phi_{1}\left[u_{\{1,2,3\}}\right]+\phi_{2}\left[u_{\{1,2,3\}}\right]+$ $\phi_{3}\left[u_{\{1,2,3\}}\right]=p_{1} p_{2}$ we find $\phi_{3}\left[u_{\{1,2,3\}}\right]=p_{1} p_{2}$, and something analogous occurs for the remaining coalitions of the list. Finally, for $\{3,4\},\{1,3,4\},\{2,3,4\}$ and $\mathrm{N}$ we find $\phi_{i}\left[u_{T}\right]=0$ for all $i \in N$. Here we are exclusively applying, as in the proof of Theorem 4.6, linearity (of course), positivity, the dummy player property, the $\mathbf{p}-$ multinomial total power property, and the property of $\mathbf{p}$-weighted payoffs for unanimity games.
} 
Hence, the profile we are looking for must be of the form $\mathbf{p}=(0.3 \mu, 0.8 \mu, \mu)$ for some $\mu \in[0,1]$. Further, by imposing the $\mathbf{p}$-multinomial total power property, we find that it holds if and only if $\mu=1$. Then, the given probabilistic value $\phi$ is the multinomial value $\lambda^{\mathbf{p}}$ defined by profile $\mathbf{p}=(0.3,0.8,1)$. Nevertheless, Theorem 4.12 will provide an alternative and easier procedure.

\subsection{Collective characterization of multinomial values}

Among semivalues, the binomial family is characterized by the monotonicity of the weighting coefficients: as was mentioned in [2], a semivalue $\psi$ on $\mathcal{G}_{N}$ is binomial if and only if its weighting coefficients $\left\{p_{s}\right\}_{s=0}^{n-1}$ are in geometric progression, i.e. satisfy, for some $\mu$, the condition $p_{s+1}=\mu p_{s}$ for $s=0,1,2, \ldots, n-2$ (maybe the simplest form of monotonicity). Strictly speaking, the condition is as follows: (i) $p_{s+1}=\mu p_{s}$ for all $s$ or (ii) $p_{s}=\mu^{\prime} p_{s+1}$ for all $s{ }^{4}$ The extension is not at all straightforward and will be given by Theorem 4.12. To this end, we need to consider two special types of players with regard to the weighting coefficients of a probabilistic value.

Definition 4.9 Let $\phi$ be a probabilistic value on $\mathcal{G}_{N}$ with weighting coefficients $\left\{p_{S}^{i}\right\}$.

- A player $h \in N$ is a $\phi$-ordinary player ${ }^{5}$ if there is $\mu_{h} \geq 0$ such that, for all $i \in N, p_{S}^{i}=$ $\mu_{h} p_{S \backslash\{h\}}^{i}$ whenever $h \in S \subseteq N \backslash\{i\}$.

- A player $h \in N$ is a $\phi$-magnetic player if $p_{S \backslash\{h\}}^{i}=0$ whenever $h \in S \subseteq N \backslash\{i\}$. This condition is equivalent to saying that $p_{S}^{i}=0$ for all $S \subseteq N \backslash\{i, h\}$.

These two notions require further detailed explanation before we proceed to use them.

Examples 4.10 (a) For the Banzhaf value $\beta$, all players are ordinary, with $\mu_{h}=1$ for all of them. The same happens for every binomial semivalue $\psi^{q}$, with $\mu_{h}=q /(1-q)$, with just one exception: for the marginal index $\psi^{1}$ all players are magnetic.

(b) The Shapley value $\varphi$ does not admit magnetic players. For $n=2$ both players are ordinary, with $\mu_{h}=1$. For $n>2$, there are no ordinary players.

(c) Let $n=3$ and assume that, for a given probabilistic value $\phi$, players 1 and 2 are ordinary and player 3 is magnetic. Then, we have, for some $\mu_{1}, \mu_{2} \geq 0$,

$$
\begin{array}{lllll}
p_{\emptyset}^{1}=0 & \stackrel{\mu_{2}}{\longrightarrow} p_{\{2\}}^{1}=\mu_{2} p_{\emptyset}^{1}=0, & p_{\{3\}}^{1} & \stackrel{\mu_{2}}{\longrightarrow} & p_{\{2,3\}}^{1}=\mu_{2} p_{\{3\}}^{1}, \\
p_{\emptyset}^{2}=0 & \stackrel{\mu_{1}}{\longrightarrow} \quad p_{\{1\}}^{2}=\mu_{1} p_{\emptyset}^{2}=0, & p_{\{3\}}^{2} & \stackrel{\mu_{1}}{\longrightarrow} & p_{\{1,3\}}^{2}=\mu_{1} p_{\{3\}}^{2}, \\
p_{\emptyset}^{3} \quad \stackrel{\mu_{1}}{\longrightarrow} p_{\{1\}}^{3}=\mu_{1} p_{\emptyset}^{3}, & p_{\{1\}}^{3} & \stackrel{\mu_{2}}{\longrightarrow} & p_{\{1,2\}}^{3}=\mu_{2} p_{\{1\}}^{3}, \\
p_{\emptyset}^{3} \quad \stackrel{\mu_{2}}{\longrightarrow} \quad p_{\{2\}}^{3}=\mu_{2} p_{\emptyset}^{3}, & p_{\{2\}}^{3} & \stackrel{\mu_{1}}{\longrightarrow} & p_{\{1,2\}}^{3}=\mu_{1} p_{\{2\}}^{3} .
\end{array}
$$

Eq. (2) gives $p_{\{3\}}^{1}, p_{\{3\}}^{2}, p_{\emptyset}^{3}>0$ and yields these relevant weighting coefficients in terms of $\mu_{1}, \mu_{2}$ :

$$
p_{\{3\}}^{1}=\frac{1}{1+\mu_{2}}, \quad p_{\{3\}}^{2}=\frac{1}{1+\mu_{1}}, \quad p_{\emptyset}^{3}=\frac{1}{1+\mu_{1}+\mu_{2}+\mu_{1} \mu_{2}} .
$$

\footnotetext{
${ }^{4}$ The dictatorial index $\psi^{0}$ satisfies (i) only, with $p_{0}=1$ and $\mu=0$. The marginal index $\psi^{1}$ satisfies (ii) only, with $p_{n-1}=1$ and $\mu^{\prime}=0$. Any other binomial semivalue, with $q \neq 0,1$, satisfies (i) and (ii) because $\mu=\frac{1-q}{q} \neq 0$; thus, $q=\frac{\mu}{1+\mu}$ and $p_{0}=\frac{1}{(1+\mu)^{n-1}}$.

${ }^{5}$ We use this term to emphasize that exceptionality corresponds to the next option, that of magnetic player.
} 
Hence, for such a value, $p_{\emptyset}^{3}=p_{\{3\}}^{1} p_{\{3\}}^{2}$. Thus, choosing arbitrarily $p_{\{3\}}^{1}=\alpha$ and $p_{\{3\}}^{2}=\beta$ (with $0<\alpha, \beta<1$ ), we find

$$
p_{\emptyset}^{3}=\alpha \beta, \quad \mu_{1}=\frac{1-\beta}{\beta}, \quad \mu_{2}=\frac{1-\alpha}{\alpha}
$$

and the remaining weighting coefficients:

$$
\begin{array}{llll}
p_{\emptyset}^{1}=0, & p_{\{2\}}^{1}=0, & p_{\{3\}}^{1}=\alpha, & p_{\{2,3\}}^{1}=1-\alpha, \\
p_{\emptyset}^{2}=0, & p_{\{1\}}^{2}=0, & p_{\{3\}}^{2}=\beta, & p_{\{1,3\}}^{2}=1-\beta, \\
p_{\emptyset}^{3}=\alpha \beta, & p_{\{1\}}^{3}=\alpha(1-\beta), & p_{\{2\}}^{3}=(1-\alpha) \beta, & p_{\{1,2\}}^{3}=(1-\alpha)(1-\beta) .
\end{array}
$$

Therefore, we have found all multinomial values having 1 and 2 as ordinary players and 3 as a magnetic player. They form a two-parametric family (with $0<\alpha, \beta<1$ ), and the profile defining each one of them is of the form $\mathbf{p}=(1-\beta, 1-\alpha, 1)$.

Remarks 4.11 (a) The conditions of Definition 4.9 are incompatible. If there were a simultaneously ordinary and magnetic player $h$ then, for any other $i \in N$, we would have $p_{S}^{i}=0$ for all $S \subseteq N \backslash\{i\}$, contradicting that these coefficients sum up to 1 .

(b) The condition of ordinary player means that the relation between $p_{S}^{i}$ and $p_{S \backslash\{h\}}^{i}$ follows a pattern common to all $i \in N$ and very similar to the monotonicity in the binomial semivalue case, although the proportionality factor depends here on player $h$ (cf. footnote 4 ).

(c) Instead, the existence of a magnetic player $h$ implies that none of the other players would join a coalition excluding $h$.

Theorem 4.12 (Collective characterization of all multinomial values). A probabilistic value $\phi$ on $\mathcal{G}_{N}$ is a multinomial value if and only if all players $h \in N$ are $\phi$-ordinary or $\phi$-magnetic. In this case, $\phi=\lambda^{\mathbf{p}}$, where $\mathbf{p}=\left(p_{1}, p_{2}, \ldots, p_{n}\right)$ is given by

$$
p_{h}= \begin{cases}\frac{\mu_{h}}{1+\mu_{h}}\left(\text { for some } \mu_{h} \geq 0\right) & \text { if } h \text { is a } \phi \text {-ordinary player } \\ 1 & \text { if } h \text { is a } \phi \text {-magnetic player. }\end{cases}
$$

Proof. $(\Rightarrow)$ Let $\phi=\lambda^{\mathbf{p}}$ for some profile p. Using Eq. (6), if $h \in S \subseteq N \backslash\{i\}$ then

$$
p_{S}^{i}=p_{h} \prod_{j \in S \backslash\{h\}} p_{j} \prod_{\substack{k \in N \backslash S: \\ k \neq i}}\left(1-p_{k}\right) \quad \text { and } \quad p_{S \backslash\{h\}}^{i}=\left(1-p_{h}\right) \prod_{j \in S \backslash\{h\}} p_{j} \prod_{\substack{k \in N \backslash S: \\ k \neq i}}\left(1-p_{k}\right),
$$

so $\left(1-p_{h}\right) p_{S}^{i}=p_{h} p_{S \backslash\{h\}}^{i}$. If $p_{h}<1$ then $p_{S}^{i}=\mu_{h} p_{S \backslash\{h\}}^{i}$, where $\mu_{h}=\frac{p_{h}}{1-p_{h}}$, and $h$ is $\lambda^{\mathbf{p}}$-ordinary. If $p_{h}=1$ then $p_{S \backslash\{h\}}^{i}=0$ and $h$ is a $\lambda^{\mathbf{p}}$-magnetic player.

$(\Leftarrow)$ Let $\phi$ be a probabilistic value such that every player is $\phi$-ordinary or $\phi$-magnetic. ${ }^{6}$ If all $h \in N$ are magnetic then, for all $i \in N$, we have $p_{S}^{i}=0$ for all $S \subset N \backslash\{i\}$ and $p_{N \backslash\{i\}}^{i}=1$ by Eq. (2), so $\phi=\lambda^{\mathbf{p}}$ where $\mathbf{p}=(1,1, \ldots, 1)$, and $\phi$ is the marginal index $\psi^{1}$. Else, let $K \subseteq N$ be the nonempty set of $\phi$-ordinary players, and hence $N \backslash K$ be the set of $\phi-$ magnetic players.

\footnotetext{
${ }^{6}$ Some points of the argument that follows are well illustrated by e.g. the particular case $n=4$.
} 
If $i \in K$ and $S \subseteq N \backslash\{i\}$ is such that $N \backslash K \nsubseteq S$, then $p_{S}^{i}=0$. The remaining weighting coefficients satisfy

$$
\sum_{\substack{S \subseteq N \backslash\{i\}: \\ N \backslash K \subseteq S}} p_{S}^{i}=1, \quad \text { where } \quad p_{S}^{i}=p_{N \backslash K}^{i} \prod_{h \in S \cap K} \mu_{h} \quad \text { for all such } S .
$$

If $j \in N \backslash K$ and $S \subseteq N \backslash\{j\}$ is such that $N \backslash(K \cup\{j\}) \nsubseteq S$, then $p_{S}^{j}=0$. The remaining weighting coefficients satisfy

$$
\sum_{\substack{S \subseteq N \backslash\{j\}: \\ N \backslash(K \cup\{j\}) \subseteq S}} p_{S}^{j}=1, \quad \text { where } \quad p_{S}^{j}=p_{N \backslash(K \cup\{j\})}^{j} \prod_{h \in S \cap K} \mu_{h} \quad \text { for all such } S .
$$

Therefore, if $i \in K$ and $j \in N \backslash K$ then

$$
p_{N \backslash K}^{i} \sum_{\substack{S \subseteq N \backslash\{i\}: \\ N \backslash K \subseteq S}} \prod_{h \in S \cap K} \mu_{h}=1 \quad \text { and } \quad p_{N \backslash(K \cup\{j\})}^{j} \sum_{\substack{S \subseteq N \backslash\{j\}: \\ N \backslash(K \cup\{j\}) \subseteq S}} \prod_{h \in S \cap K} \mu_{h}=1 .
$$

In both cases, the sum is of the form

$$
1+\sum_{r \in L} \mu_{r}+\sum_{\substack{r, s \in L: \\ r<s}} \mu_{r} \mu_{s}+\sum_{\substack{r, s, t \in L: \\ r<s<t}} \mu_{r} \mu_{s} \mu_{t}+\cdots+\prod_{r \in L} \mu_{r},
$$

with $L=K \backslash\{i\}$ in case of $i$ and $L=K$ in case of $j$.

Now we define a tendency profile $\mathbf{p}=\left(p_{1}, p_{2}, \ldots, p_{n}\right)$ by setting

$$
p_{h}=\left\{\begin{array}{lll}
\frac{\mu_{h}}{1+\mu_{h}} & \text { if } & h \in K \\
1 & \text { if } \quad h \in N \backslash K .
\end{array}\right.
$$

Thus, $p_{h}<1$ and hence $\mu_{h}=\frac{p_{h}}{1-p_{h}}$ makes sense for each $h \in K$. By replacing each $\mu_{k}$ in terms of $p_{k}$ for each $k \in L$, it is not difficult to verify that the reciprocal of the sum given in Eq. (11) is

$$
\frac{\prod_{k \in L}\left(1-p_{k}\right)}{\sum_{S \subseteq L} \prod_{k \in S} p_{k} \prod_{\ell \in L \backslash S}\left(1-p_{\ell}\right)}
$$

but the denominator equals 1 since the partial derivatives of the multilinear function

$$
f\left(\left(x_{k}\right)_{k \in L}\right)=\sum_{S \subseteq L} \prod_{k \in S} x_{k} \prod_{\ell \in L \backslash S}\left(1-x_{\ell}\right)
$$

vanish and $f(1,1, \ldots, 1)=1$. Thus, $p_{N \backslash K}^{i}$ and $p_{N \backslash(K \cup\{j\})}^{j}$ are of the desired form since, as $p_{\ell}=1$ if $\ell \in N \backslash K$, we can write

$$
p_{N \backslash K}^{i}=\prod_{k \in K \backslash\{i\}}\left(1-p_{k}\right) \prod_{\ell \in N \backslash K} p_{\ell} \quad \text { and } \quad p_{N \backslash(K \cup\{j\})}^{j}=\prod_{k \in K}\left(1-p_{k}\right) \prod_{\substack{\ell \in N \backslash K: \\ \ell \neq j}} p_{\ell} .
$$


Finally, for any $S$ such that $N \backslash K \subseteq S \subseteq N \backslash\{i\}$,

$$
p_{S}^{i}=p_{N \backslash K}^{i} \prod_{h \in S \cap K} \frac{p_{h}}{1-p_{h}}=\prod_{h \in S} p_{h} \prod_{\substack{\ell \in N \backslash S: \\ \ell \neq i}}\left(1-p_{\ell}\right)
$$

and, for any $S$ such that $N \backslash(K \cup\{j\}) \subseteq S \subseteq N \backslash\{j\}$,

$$
p_{S}^{j}=p_{N \backslash(K \cup\{j\})}^{j} \prod_{h \in S \cap K} \frac{p_{h}}{1-p_{h}}=\prod_{h \in S} p_{h} \prod_{\substack{\ell \in N \backslash S: \\ \ell \neq j}}\left(1-p_{\ell}\right) .
$$

Then $\phi=\lambda^{\mathbf{p}}$ for the profile $\mathbf{p}$ defined above.

Corollary 4.13 As a consequence of Theorem 4.12, it immediately follows that, if $\phi=\lambda^{\mathbf{P}}$ is a multinomial value, then player $h \in N$ is $\phi$-ordinary if $p_{h}<1$, and $\phi$-magnetic if $p_{h}=1$.

Remark 4.14 The difference between monotonicity at individual level established in Theorem 4.12 and uniform monotonicity that characterizes binomial semivalues is a new sample of the higher versatility of the multinomial values. Moreover, Theorem 4.12 provides an easy procedure to check whether a probabilistic value is a multinomial value and determine, if it is so, the tendency profile that defines it - cf. Example 4.10(c) above and Example 4.15(c) below.

Examples 4.15 (a) The Shapley value is multinomial only for $n=2$. In fact, in this case $\varphi$ and $\beta$ coincide. Thus, Example 4.10(b) does not contradict the theorem.

(b) According to Theorem 4.12, the (two-parametric family of) values obtained in Example 4.10 (c) are all multinomial by the simple fact of having only ordinary or magnetic players. Their individual description given in that example is also possible from Theorem 4.12.

(c) Let $\phi$ be the probabilistic value for $n=3$ defined by the weighting coefficients

$$
\begin{array}{llll}
p_{\emptyset}^{1}=0, & p_{\{2\}}^{1}=0, & p_{\{3\}}^{1}=0.2, & p_{\{2,3\}}^{1}=0.8, \\
p_{\emptyset}^{2}=0, & p_{\{1\}}^{2}=0, & p_{\{3\}}^{2}=0.8, & p_{\{1,3\}}^{2}=0.2, \\
p_{\emptyset}^{3}=0.4, & p_{\{1\}}^{3}=0.1, & p_{\{2\}}^{3}=0.4, & p_{\{1,2\}}^{3}=0.1 .
\end{array}
$$

It is easy to check that, with regard to $\phi$, player 1 is ordinary (with $\mu_{1}=1 / 4$ ) and player 3 is magnetic, but player 2 is neither ordinary nor magnetic. Then, using Theorem 4.12 again, $\phi$ is not a multinomial value.

\section{An example of application to simple games}

As was mentioned in Remark 3.2(f), the calculation of a value in terms of the multilinear extension of the game was extended to any multinomial probabilistic value $[18,12]$ : if $\lambda^{\mathbf{p}}$ is such a value and $f$ is the multilinear extension of game $v \in \mathcal{G}_{N}$ then

$$
\lambda_{i}^{\mathbf{p}}[v]=\frac{\partial f}{\partial x_{i}}\left(p_{1}, p_{2}, \ldots, p_{n}\right) \quad \text { for each } i \in N .
$$

We begin by introducing a bit more of notation and giving a preliminary result. If $v \in \mathcal{G}_{N}$, its multilinear extension $f$ is, in principle, defined on the whole Euclidean space $\mathbb{R}^{n}$ although, generally, 
we are only interested in its behavior in the $n$-cube $[0,1]^{n}$. The set $2^{N}$ of coalitions of $N$ can be identified with $\{0,1\}^{n}$, the set of vertices of the cube, through the map $S \mapsto \mathbf{x}^{S}=\left(x_{1}^{S}, x_{2}^{S}, \ldots, x_{n}^{S}\right)$ given by $x_{i}^{S}=1$ if $i \in S$ or else $x_{i}^{S}=0$. Then, $v(S)=f\left(\mathbf{x}^{S}\right)$ for all $S \subseteq N$. We shall use in the sequel, for any $\mathbf{x}=\left(x_{1}, x_{2}, \ldots, x_{n}\right) \in \mathbb{R}^{n}$, notation like e.g.

$$
f\left(1_{i}, \mathbf{x}\right)=f\left(x_{1}, \ldots, \stackrel{i}{1}, \ldots, x_{n}\right) .
$$

Proposition 5.1 (Domènech et al. [8]) Let $f$ be the multilinear extension of game $v \in \mathcal{G}_{N}$.

(a) For each $i \in N, f$ is a linear function of $x_{i}$, that is, $f(\mathbf{x})=a+b x_{i}$ where $a=f\left(0_{i}, \mathbf{x}\right)$ and $b=f\left(1_{i}, \mathbf{x}\right)-f\left(0_{i}, \mathbf{x}\right)$ for all $\mathbf{x}$. Then

$$
f(\mathbf{x})=x_{i} f\left(1_{i}, \mathbf{x}\right)+\left(1-x_{i}\right) f\left(0_{i}, \mathbf{x}\right) \text { for all } \mathbf{x} .
$$

(b) Now, let $\mathbf{p}$ be a profile on $N$. For all $i \in N$,

$$
\lambda_{i}^{\mathbf{p}}[v]=\frac{\partial f}{\partial x_{i}}(\mathbf{p})=f\left(1_{i}, \mathbf{p}\right)-f\left(0_{i}, \mathbf{p}\right) .
$$

(c) Moreover,

$$
\left(1-p_{i}\right) \lambda_{i}^{\mathbf{p}}[v]=f\left(1_{i}, \mathbf{p}\right)-f(\mathbf{p})
$$

From now on we will focus on simple games. Parliamentary bodies provide conspicuous examples. If voting discipline within parties holds, then the agents are the parties, and each party controls the votes of all its representatives. Otherwise, each parliamentarian is an agent with one vote (symmetric or $k$-out-of-n game). The US Senate can be viewed in this second way due to the political freedom to act that each senator enjoys. Other examples where the agents are, actually, individuals with one vote each are popular juries or the Constitutional Court.

In this context, if $p_{i}$ is the probability of each player $i$ to vote for the proposal (and hence $1-p_{i}$ is the probability to vote against or abstain), $f(\mathbf{p})=f\left(p_{1}, \ldots, p_{n}\right)$ yields the probability of the proposal to be socially accepted and

$$
f\left(1_{i}, \mathbf{p}\right)-f(\mathbf{p})=\left(1-p_{i}\right) \lambda_{i}^{\mathbf{p}}[v]
$$

can be viewed as the increment on the probability of acceptance of the proposal when only player $i$ changes his probability of voting yes from $p_{i}$ to 1 and becomes therefore a magnetic player for the new multinomial value arising after the change of tendency of this player. This proves the close relationship between the multinomial values on simple games and the potential decisiveness index $\Omega^{\mathbf{p}}$ introduced by Freixas and Pons [13] and defined, for each $i \in N$, by

$$
\Omega_{i}^{\mathbf{p}}[v]=f\left(1_{i}, \mathbf{p}\right)-f(\mathbf{p}) .
$$

This shows the precise way in which the increase of the acceptance of the proposal depends on the multinomial value $\lambda_{i}^{\mathbf{p}}[v]$. Taking into account this fact, we can use multinomial values to determine which player has more influence in order to change the result of the voting process when he ensures his positive vote. In other words, in situations of corruption, these values play an important role in order to determine which are the players that may cause, by turning into a magnetic player, the maximum increase in the probability of passing the proposal, and are therefore the best candidates to be bribed.

Example 5.2 (Example 1.1 revisited) The simple game $v$ associated to Example 1.1 is defined in $N=\{1,2,3,4,5\}$, where players 1,2 and 3 are the founding members, by the family of its winning coalitions

$$
W(v)=\{\{1,2,3\},\{1,2,3,4\},\{1,2,3,5\},\{1,2,3,4,5\}\} .
$$


Notice that $v=u_{\{1,2,3\}}$. We will compute $\lambda^{\mathbf{p}}[v]$ for any profile $\mathbf{p}$. The multilinear extension of $v$ is

$$
f\left(x_{1}, x_{2}, x_{3}, x_{4}, x_{5}\right)=x_{1} x_{2} x_{3}
$$

The calculation of $\lambda^{\mathbf{P}}[v]$ derives from Eq. (12):

$$
\begin{aligned}
\lambda_{1}^{\mathbf{p}}[v] & =p_{2} p_{3}, \\
\lambda_{2}^{\mathbf{p}}[v] & =p_{1} p_{3}, \\
\lambda_{3}^{\mathbf{p}}[v] & =p_{1} p_{2}, \\
\lambda_{4}^{\mathbf{p}}[v] & =\lambda_{5}^{\mathbf{p}}[v]=0 .
\end{aligned}
$$

Notice that the introduction of tendency profiles breaks the symmetry between players 1,2 and 3. Nevertheless, a sort of "structural" symmetry still exists between $\lambda_{1}^{\mathbf{p}}[v], \lambda_{2}^{\mathbf{p}}[v]$ and $\lambda_{3}^{\mathbf{p}}[v]$ since, for instance, $\lambda_{2}^{\mathbf{p}}[v]$ is obtained from $\lambda_{1}^{\mathbf{p}}[v]$ by replacing $p_{2}$ with $p_{1}$. This is due to the symmetric positions of each pair of players in the game, which translates to the multilinear extension.

Now we will answer question (a) in Example 1.1. To this end, it suffices to compare $\lambda_{1}^{\mathbf{p}}[v], \lambda_{2}^{\mathbf{p}}[v]$ and $\lambda_{3}^{\mathbf{p}}[v]$. If $i, j, k$ denote the three distinct nonnull players, we have for all of them

$$
\lambda_{i}^{\mathbf{p}}[v]-\lambda_{j}^{\mathbf{p}}[v]=p_{k}\left(p_{j}-p_{i}\right) \geq 0 \quad \Longleftrightarrow \quad p_{j} \geq p_{i} .
$$

For example, if $p_{1}=p_{2}>p_{3}$ then $\lambda_{1}^{\mathbf{p}}[v]=\lambda_{2}^{\mathbf{p}}[v]<\lambda_{3}^{\mathbf{p}}[v]$, so player 3 has the greatest decision power.

(b) In order to know which member of firm A must be bribed for firm B we need to consider $\Omega_{i}^{\mathbf{p}}[v]=f\left(1_{i}, \mathbf{p}\right)-f(\mathbf{p})=\left(1-p_{i}\right) \lambda_{i}^{\mathbf{p}}[v]$ for $i=1,2$ and 3 . It is easy to see that, if $i, j, k$ denote these players,

$$
\Omega_{i}^{\mathbf{p}}[v]-\Omega_{j}^{\mathbf{p}}[v]=p_{k}\left(p_{j}-p_{i}\right)=\lambda_{i}^{\mathbf{p}}[v]-\lambda_{j}^{\mathbf{p}}[v]
$$

and then, if $i \neq j$, member $i$ is better than member $j$ to be bribed if and only if $p_{j}>p_{i}$.

If we consider a particular profile, e.g. of the form $\left(p, 1-p, p, p_{4}, p_{5}\right)$, with $p, p_{4}, p_{5} \in[0,1]$, we obtain

$$
\begin{aligned}
\lambda_{1}^{\mathbf{p}}[v] & =(1-p) p \\
\lambda_{2}^{\mathbf{p}}[v] & =p^{2}, \\
\lambda_{3}^{\mathbf{p}}[v] & =p(1-p), \\
\lambda_{4}^{\mathbf{p}}[v] & =\lambda_{5}^{\mathbf{p}}[v]=0 .
\end{aligned}
$$

We can compare $\lambda_{1}^{\mathbf{p}}[v]$ and $\lambda_{2}^{\mathbf{p}}[v]$ in order to know which is the best candidate to be bribed.

$$
\lambda_{1}^{\mathbf{p}}[v] \geq \lambda_{2}^{\mathbf{p}}[v] \quad \Longleftrightarrow \quad p \leq 1 / 2
$$

so player 1 (as well as player 3 ) is the best candidate to be bribed iff $p \leq \frac{1}{2}$. If member 1 is bribed and turns therefore into a magnetic player, a new profile $\left(1,1-p, p, p_{4}, p_{5}\right)$ arises and, in this new situation, according to Remark 3.2(c), if $1 \notin S$ then $p_{S}^{i}=0$ for any $i \notin S$. This means that no other member is interested in joining a coalition not including member 1 . If $p<\frac{1}{2}$, under the new profile member 3 has more decision power than member 2 since $1-p>p$.

Remark 5.3 (a) More generally, if $N=\{1,2, \ldots, n\}$ and $v=u_{S}$ for some nonempty $S \subseteq N$, to study players' decisiveness $\lambda_{i}^{\mathbf{p}}[v]$ is equivalent to study players' potential decisiveness $\Omega_{i}^{\mathbf{p}}[v]=$ $\left(1-p_{i}\right) \lambda_{i}^{\mathbf{p}}[v]$. Indeed, for such a game,

$$
f\left(x_{1}, x_{2}, \ldots, x_{n}\right)=\prod_{i \in S} x_{i}, \quad \lambda_{i}^{\mathbf{p}}[v]=\prod_{k \in S \backslash\{i\}} p_{k}
$$


and, for all $i, j \in S$,

$$
\lambda_{i}^{\mathbf{p}}[v]-\lambda_{j}^{\mathbf{p}}[v]=\left(p_{j}-p_{i}\right) \prod_{k \in S \backslash\{i, j\}} p_{k}=\Omega_{i}^{\mathbf{p}}[v]-\Omega_{j}^{\mathbf{p}}[v] .
$$

(b) A similar situation occurs if $N=\{1,2, \ldots, n\}$ and the family of minimal winning coalitions of the game is $W^{m}(v)=\{S, N \backslash S\}$ for some nonempty $S \subset N$. In this case,

$$
f\left(x_{1}, x_{2}, \ldots, x_{n}\right)=\prod_{i \in S} x_{i}+\prod_{j \in N \backslash S} x_{j}-\prod_{k \in N} x_{k}
$$

and, for all $i, j \in S$, we have

$$
\lambda_{i}^{\mathbf{p}}[v]-\lambda_{j}^{\mathbf{p}}[v]=\prod_{k \in S \backslash\{i, j\}} p_{k}\left(1-\prod_{\ell \in N \backslash S} p_{\ell}\right)\left(p_{j}-p_{i}\right)=\Omega_{i}^{\mathbf{p}}[v]-\Omega_{j}^{\mathbf{p}}[v],
$$

whereas, for all $i, j \in N \backslash S$,

$$
\lambda_{i}^{\mathbf{p}}[v]-\lambda_{j}^{\mathbf{p}}[v]=\prod_{\substack{k \in N \backslash S: \\ k \neq i, j}} p_{k}\left(1-\prod_{\ell \in S} p_{\ell}\right)\left(p_{j}-p_{i}\right)=\Omega_{i}^{\mathbf{p}}[v]-\Omega_{j}^{\mathbf{p}}[v] .
$$

\section{Acknowledgments}

The authors wish to thank two anonymous reviewers for their helpful comments.

\section{References}

[1] Amer R. and Giménez J.M. [2003]: "Modification of semivalues for games with coalition structures." Theory and Decision 54, 185-205.

[2] Alonso, J.M., Carreras, F. and Puente, M.A. [2007]: "Axiomatic characterizations of the symmetric coalitional binomial semivalues." Discrete Applied Mathematics 155, 2282-2293.

[3] Carreras, F. [2004]: " $\alpha$-decisiveness in simple games." Theory and Decision 56, 77-91. Also in: Essays on Cooperative Games (G. Gambarelli, ed.), Kluwer Academic Publishers, 77-91.

[4] Carreras, F. [2005]: “A decisiveness index for simple games." European Journal of Operational Research 163, 370-387.

[5] Carreras, F. and Puente, M.A. [2012]: "Symmetric coalitional binomial semivalues." Group Decision and Negotiation 21, 637-662.

[6] Carreras, F. and Puente, M.A. [2015a]: "Multinomial probabilistic values." Group Decision and Negotiation 24, 981-991.

[7] Carreras, F. and Puente, M.A. [2015b]: "Coalitional multinomial probabilistic values." European Journal of Operational Research 245, 236-246.

[8] Domènech, M., Giménez, J.M. and Puente, M.A. [2016]: "Some properties for probabilistic and multinomial (probabilistic) values on cooperative games." Optimization 65, 1377-1395. 
[9] Dubey, P. and Shapley, L.S. [1979]: "Mathematical properties of the Banzhaf power index." Mathematics of Operations Research 4, 99-131.

[10] Dubey, P., Neyman, A. and Weber, R.J. [1981]: "Value theory without efficiency." Mathematics of Operations Research 6, 122-128.

[11] Feltkamp, V. [1995]: "Alternative axiomatic characterizations of the Shapley and Banzhaf values." International Journal of Game Theory 24, 179-186.

[12] Freixas, J. and Puente, M.A. [2002]: "Reliability importance measures of the components in a system based on semivalues and probabilistic values." Annals of Operations Research 109, $331-342$.

[13] Freixas, J. and Pons, M. [2015]: "An axiomatic characterization of the potential decisiveness index." Journal of the Operational Research Society 66, 353-359.

[14] Giménez J.M., Llongueras, M.D. and Puente, M.A. [2014]: "Partnership formation and multinomial values." Discrete Applied Mathematics 170, 7-20.

[15] Owen, G. [1972]: "Multilinear extensions of games." Management Science 18, 64-79.

[16] Owen, G. [1975]: "Multilinear extensions and the Banzhaf value." Naval Research Logistics Quarterly 22, 741-750.

[17] Owen, G. [1978]: "Characterization of the Banzhaf-Coleman index." SIAM Journal of Applied Mathematics 35, 315-327.

[18] Puente, M.A. [2000]: Contributions to the representability of simple games and to the calculus of solutions for this class of games (in Spanish). Ph.D. Thesis. Universitat Politècnica de Catalunya, Spain.

[19] Shapley, L.S. [1953]: "A value for n-person games." In: Contributions to the Theory of Games II (H.W. Kuhn and A.W. Tucker, eds.), Princeton University Press, Annals of Mathematical Studies 28, 307-317.

[20] Weber, R.J. [1988]: "Probabilistic values for games." In: The Shapley Value: Essays in Honor of Lloyd S. Shapley (A.E. Roth, ed.), Cambridge University Press, 101-119. 